\title{
Surgical Management of Retraction Pockets: Does Mastoidectomy have a Role?
}

\author{
Francesco Dispenza ${ }^{1}$ Antonina Mistretta ${ }^{1}$ Federico Gullo ${ }^{1}$ Francesco Riggio ${ }^{1}$ Francesco Martines ${ }^{2}$ \\ 1 Department of Otolaryngology, University of Palermo, Palermo, PA, Italy \\ 2 Department of Audiology, University of Palermo, Palermo, PA, Italy \\ Address for correspondence Dispenza Francesco, MD, U.O. \\ Otorinolaringoiatria, University of Palermo, via Oreto 339, Palermo, \\ PA, 90124, Italy (e-mail: francesco.dispenza@gmail.com).
} Int Arch Otorhinolaryngol 2021;25(1):e12-e17.

\begin{abstract}
Introduction Retraction pocket is a condition in which the eardrum lies deeper within the middle ear. Its management has no consensus in literature.

Objective To assess the role of mastoidectomy in the management of retraction pockets added to a tympanoplasty.

Methods Prospective study of patients with retraction pocket and referred to surgery. The patients were randomly assigned to two groups: one managed with tympanoplasty and mastoidectomy and the other group with tympanoplasty only. The minimum follow-up considered was 12 months. The outcomes were: integrity of eardrum, recurrence, and hearing status.

Results This study included 43 patients. In 24 cases retraction occurred in the posterior half of the eardrum, and in 19 patients there was clinical evidence of ossicular interruption. The two groups of treatment were composed by: 21 patients that underwent tympanoplasty with mastoidectomy and 22 patients had only tympanoplasty. One case of the first group had a recurrence. In 32 cases patients follow up was

Keywords

- tympanic membrane

- mastoidectomy

- tympanoplasty

- middle ear

- ossiculoplasty

- otitis media longer than 48 months. The average air-bone gap changed from $22.1 \mathrm{~dB}$ to $5 \mathrm{~dB}$. The percentage of air-bone gap improvement was assessed at $60 \%$ in those patients treated with mastoidectomy, and $64.3 \%$ in those without it $(p>0.5)$.

Conclusion Tympanoplasty and ossiculoplasty should be considered to treat atelectatic middle ear and ossicular chain interruption. Mastoidectomy as a way to increase air volume in the ear seems to be a paradox; it does not add favorable prognostic factor to management of retraction pockets.
\end{abstract}

\section{Introduction}

Tympanic membrane retraction refers to a condition in which a portion or the whole eardrum lies deeper within the middle ear than its normal position, as a result of negative pressure within the middle ear cleft, because of impaired airflow to this space. A retracted segment of the tympanic membrane is known as retraction pocket (RP). The eardrum is made up of two parts: the pars tensa and the pars flaccida. Both of these parts may become retracted (together as well). Commonly, the tympanic membrane retractions are asymptomatic, especially in the early phase, so they can be discovered during a simple ear examination. On the other hand, atelectasis of the middle ear may cause conductive hearing loss by restricting soundinduced vibrations of the tympanic membrane and ossicular chain as well. Other symptoms, such as otorrhea, could be received

April 10, 2019

accepted

February 28, 2020

published online

April 24, 2020
DOI https://doi.org/ 10.1055/s-0040-1709196. ISSN 1809-9777.

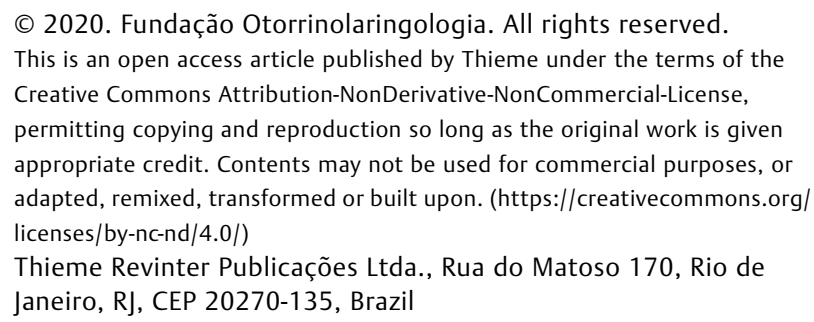


present. Adhesive otitis media is the far advanced stage of atelectasis and it has a multi-factorial pathogenesis. The knowledge of the real prevalence of RPs in any given population is not reachable, because this condition is primarily asymptomatic. In children between 5 and 16 years old, the prevalence of atelectasis of the eardrum is estimated at $\sim 14$ to $26 \%$ in the pars flaccida (attic retraction) and only 0.3 to $3.7 \%$ in the pars tensa (atrophy and retraction). ${ }^{1}$ The prevalence of RPs in adults was reported in a population sample as $9.6 \%$ in pars flaccida and $7 \%$ in pars tensa. ${ }^{2}$

Tympanic membrane retraction can be stable over time, although sometimes it can develop into cholesteatoma. Choosing the right time in treating a RP to prevent complications is difficult, and no consensus or guidelines on this issue are available. $^{3}$

The first classification of RP was made by Sadè, and it consists of four grades of severity: grade I refers to mild retraction of the tympanic membrane; grade II retraction with contact to the incudo-stapedial joint (with or without incudostapedial joint erosion); grade III retraction without adhesion of pars tensa to the promontory; grade IV adhesion to the promontory. 4

Borgstein, in 2007, made one of the latest classifications in the literature, in a study referring specifically to the pediatric population. $^{6}$

Tympanic retraction at grade I or II of Sadè can be solved without surgery; this is true especially in the case of children, in whom a spontaneous resolution is common (35\%, according with Cassano). ${ }^{7}$

Grades III and IV tympanic retractions should be surgically treated if there are other features that could lead to conductive hearing loss or cholesteatoma. According to Charachon, $16 \%$ of patients diagnosed with a retraction pocket experience a worsening of the condition within 5 years. ${ }^{8}$ According to Kasbekar, cholesteatoma lies behind $31 \%$ of eardrum retractions. ${ }^{9}$ While Cassano reported that the tendency of progression of a retracted eardrum into cholesteatoma is $\sim 20 \%{ }^{7}$

There are a variety of surgical treatments described to manage RPs. Our prospective study analyzed tympanoplasty with and without mastoidectomy, preserving the integrity of the posterior wall of the ear canal. The aim of the present study was to evaluate the role of mastoidectomy in the management of RPs in term of recurrence and hearing gain.

\section{Materials and Methods}

All patients diagnosed with RPs between 2004 and 2015 were evaluated prospectively to establish the grading and the stage. The patient's data were recorded: personal information, symptoms onset, subjective hearing issue, and any relevant medical treatment done. All patients underwent otomicroscopy, pure tone audiometry (PTA) average of $0,5-1-2-4 \mathrm{kHz}$ frequencies, tympanometry, and endoscopy of the upper airways. Those patients matching the following criteria were referred to surgery and were object of the study: grades III and IV of retraction (Sadè), marginal RP with bone erosion, non-controllable RP, non-self-cleansing RP, and recurrent otorrhea. The exclusion criterion was evidence of cholesteatoma. A comput- ed tomography (CT) scan was obtained in all patients underwent surgery. All patient undersigned an informed consent to procedure and participation to the study. Our divisional review board approved the study nr. 3-2004.

The patients were randomly assigned to two groups: the first underwent tympanoplasty plus mastoidectomy, and the second group underwent only tympanoplasty, in both groups with or without ossiculoplasty, if necessary. A simple randomization was done by a computer-generated random number with Microsoft Excel (Microsoft Corp., Redmond, WA, USA) function to address the consecutive patients to the two groups. The sample size was calculated by the formula: sample size $=($ distribution of $50 \%) /([$ margin of error $\% /$ confidence level score] squared) with confidence interval of $15 \%$ and confidence level of $95 \%$. The result in our population was 43 subjects among the residents in our city $(\sim 800,000)$.

The surgical technique applied was excision of RP and tympanic reconstruction with underlay temporalis fascia graft and tragal cartilage or fascia graft alone, the ossicular chain interruption was restored by partial or total ossiculoplasty, a simple mastoidectomy with preservation of the ear canal wall was done in those patients included in the first group. Clinical examination and medications of patients were done at $7,15,21$, and 28 postoperative days. The minimum follow-up considered was 12 months.

The outcomes were recurrent $\mathrm{RP}$ and $\mathrm{ABG}$; surgical operations were considered successful when the tympanic membrane was intact during the latest follow-up examinations, and ABG closure was considered as secondary outcome.

\section{Results}

This study followed a sample of 43 patients (-Table 1). This sample size had a confidence level of $95 \%$ and a confidence interval of $15 \%$. The mean age of the patients was 29.3 years (range 17-57). In 24 cases, retraction occurred in the posterior half of the eardrum, with adhesion to the promontory, and, in 19 patients, there was clinical evidence of ossicular chain interruption.

As - Table 2 shows, in 16 cases, the retraction occurred in the pars flaccida, of which 12 with some erosion of the ear canal bone. Only 2 patients presented anterior RP.

The average presurgical ABG of the sample was $22.1 \mathrm{~dB}$. The ABG was evidently greater in those patients with ossicular chain interruption ( $29.3 \mathrm{~dB}$ versus $12.4 \mathrm{~dB}$ ).

The 2 groups of treatment were composed by 21 patients that underwent tympanoplasty with mastoidectomy and 22 patients had only tympanoplasty.

Tympanic membrane reconstruction was done by both temporalis fascia graft and tragal cartilage in 30 patients and with fascia alone in 13 cases. There were no cases of early postoperative complications. Patients were discharged the day after the surgical treatment. In 9 cases, the ossiculoplasty was done by autologous incus reshaped and replaced (-Fig. 1). In 10 cases, the erosion was subtotal needing a titanium Kurz Variac partial prosthesis implant (Heinz Kurz GmbH Medizintechnik, Dusslingen, Germany ). 
14 Surgical Management of Retraction Pockets Dispenza et al.

Table 1 Baseline characteristics of the present series

\begin{tabular}{|c|c|c|c|c|c|c|c|c|}
\hline & & \multicolumn{2}{|c|}{ Group 1} & \multicolumn{2}{|c|}{ Group 2} & & \multicolumn{2}{|c|}{ Series } \\
\hline \multicolumn{2}{|l|}{ Mean age } & \multicolumn{2}{|c|}{$29.4(19-57)$} & \multicolumn{2}{|c|}{$28.9(17-50)$} & $p>0.5$ & \multicolumn{2}{|c|}{$\begin{array}{l}29.3(17-57) \\
\text { years }\end{array}$} \\
\hline \multicolumn{2}{|l|}{$\mathrm{M} / \mathrm{F}$ ratio } & \multicolumn{2}{|c|}{$11 / 10$} & \multicolumn{2}{|c|}{$11 / 11$} & $p>0.7$ & \multicolumn{2}{|c|}{$23 / 20$} \\
\hline \multicolumn{2}{|l|}{ Subjective tinnitus } & \multicolumn{2}{|c|}{$6(28.5 \%)$} & \multicolumn{2}{|c|}{$5(22.7 \%)$} & $p>0.7$ & \multicolumn{2}{|c|}{$11(25.5 \%)$} \\
\hline \multirow{2}{*}{\multicolumn{2}{|c|}{ Grading of RPs }} & III & IV & III & IV & \multirow[t]{2}{*}{$p>0.7$} & III & IV \\
\hline & & 10 & 11 & 10 & 12 & & 20 & 23 \\
\hline \multirow[t]{2}{*}{ RPs site } & Pars tensa & \multicolumn{2}{|c|}{$13(61.9 \%)$} & \multicolumn{2}{|c|}{$14(63.6 \%)$} & \multirow[t]{2}{*}{$p>0.7$} & \multicolumn{2}{|c|}{$27(62.8 \%)$} \\
\hline & Pars flaccida & \multicolumn{2}{|c|}{$8(38.1 \%)$} & \multicolumn{2}{|c|}{$8(36.4 \%)$} & & \multicolumn{2}{|c|}{$16(37.2 \%)$} \\
\hline \multicolumn{2}{|c|}{ Ossicular chain interruption (incus-stapes joint) } & \multicolumn{2}{|c|}{$10(47.6 \%)$} & \multicolumn{2}{|c|}{$9(40.9 \%)$} & $p>0.7$ & \multicolumn{2}{|c|}{$19(44.1 \%)$} \\
\hline \multicolumn{2}{|l|}{ Air-bone gap } & \multicolumn{2}{|c|}{$\begin{array}{l}23.4 \mathrm{~dB} \\
\text { (SD } 8.5 \mathrm{~dB})\end{array}$} & \multicolumn{2}{|c|}{$\begin{array}{l}21.7 \mathrm{~dB} \\
(\mathrm{SD} 5.1 \mathrm{~dB})\end{array}$} & $p>0.5$ & \multicolumn{2}{|c|}{$\begin{array}{l}22.1 \mathrm{~dB} \\
(\mathrm{SD} 7.7 \mathrm{~dB})\end{array}$} \\
\hline \multicolumn{2}{|l|}{ Otorrhea episodes } & \multicolumn{2}{|c|}{$4(19.0 \%)$} & \multicolumn{2}{|c|}{$4(18.1 \%)$} & $p>0.9$ & \multicolumn{2}{|c|}{$8(18.6 \%)$} \\
\hline \multirow[t]{2}{*}{ Reconstruction technique } & Fascia + cartilage & 15 & & 15( & & $p>0.7$ & 30( & \\
\hline & Fascia & $6(2$ & & $7(3$ & & & 13( & \\
\hline
\end{tabular}

Abbreviations: dB: decibels; F, female; M, male; RPs: retraction pockets; SD: standard deviation.

Table 2 Retraction pockets (RPs) findings according to site of retraction

\begin{tabular}{|l|l|l|}
\hline \multirow{2}{*}{ RP site } & Pars tensa & Pars flaccida \\
\cline { 2 - 3 } & 27 (2 anterior half) & 16 \\
\hline Ear canal erosion & 1 & 12 \\
\hline $\begin{array}{l}\text { Ossicular chain } \\
\text { interruption } \\
\text { (incus-stapes joint) }\end{array}$ & 15 & 4 \\
\hline Air-bone gap & $29.3 \mathrm{~dB}$ (SD 4.9) & $\begin{array}{l}12.4 \mathrm{~dB} \\
(\mathrm{SD} 2.9)\end{array}$ \\
\hline Otorrhea episodes & 5 & 3 \\
\hline
\end{tabular}

Abbreviations: $\mathrm{dB}$, decibels; SD, standard deviation.

In 32 patients, follow up was longer than 48 months, while it was ranged between 36 and 12 months for the other 11 patients.

The ABG for all patients changed from a preoperative average of $22.1 \mathrm{~dB}$ to a postoperative average of $5 \mathrm{~dB}$, a mean improvement of $12.5 \mathrm{~dB}$. The minimal $\mathrm{ABG}$ improvement was $5 \mathrm{~dB}$, while the best value was $30 \mathrm{~dB}$. - Tables 3 and 4 summarize all hearing results.

The first subgroup reached a postoperative average $A B G$ of $6 \mathrm{~dB}$, with an average $A B G$ improvement of $13.8 \mathrm{~dB}$. In the second subgroup, a postoperative average $A B G$ of $5.3 \mathrm{~dB}$ was obtained, with an average ABG improvement of $12.1 \mathrm{~dB}$.

Patients with ossicular chain erosion who underwent ossicular reconstruction had an $\mathrm{ABG}$ improvement of $16.7 \mathrm{~dB}$. Their postoperative average $A B G$ was $13.3 \mathrm{~dB}$. The initial condition of the ossicular chain (erosion or integrity) seems to be the most influential clinical feature to predict closure of the $A B G$.

The percentage of $A B G$ improvement was assessed at $60 \%$ in those patients treated with mastoidectomy, and $64.3 \%$ in those without it; the Chi-squared test did not show a statistically significant difference $p>0.5$.

The tympanic membrane status during follow-up was good in all patients but one, from the first subgroup, who showed long term complications (48 months) presenting a cholesteatoma with a posterior retraction pocket recurrence and perforation of the cartilage graft. In 2 patients, a small tendency to retraction (grade II) was observed at 24 months of follow-up, but the tympanic membrane was stable over time (-Fig. 2).

\section{Discussion}

Tympanoplasty should be considered an effective procedure in the treatment of RP, and, in the literature, absence of recurrence is observed in 67 to $74 \% .^{10-13}$ Before choosing whether to operate or not on a patient, the surgeon should evaluate several features of the retracted eardrum, such as: the grading of severity, features of hearing loss, the site of the eardrum involved, the status of ossicular chain, and the characteristic of the RP.

The mastoidectomy leads to increase of air in the ear "buffer system"; this serves to correct the air pressure imbalance between the external and middle ear (that is observable in most people suffering from atelectatic otitis). Unfortunately, it can create granulation tissue instead of space for gaseous exchange between air and mucosa, and it can make the surgical results worse than expected (i.e., an injury to the seventh cranial nerve) and it often does not solve $A B G$ or prevent future recurrences. ${ }^{14}$

Shew et al evaluated the management of chronic ear disease in a retrospective cohort study, and they found that tympanoplasty with concurrent mastoidectomy increases surgical time, but it is not associated with any increased postoperative complications compared with 


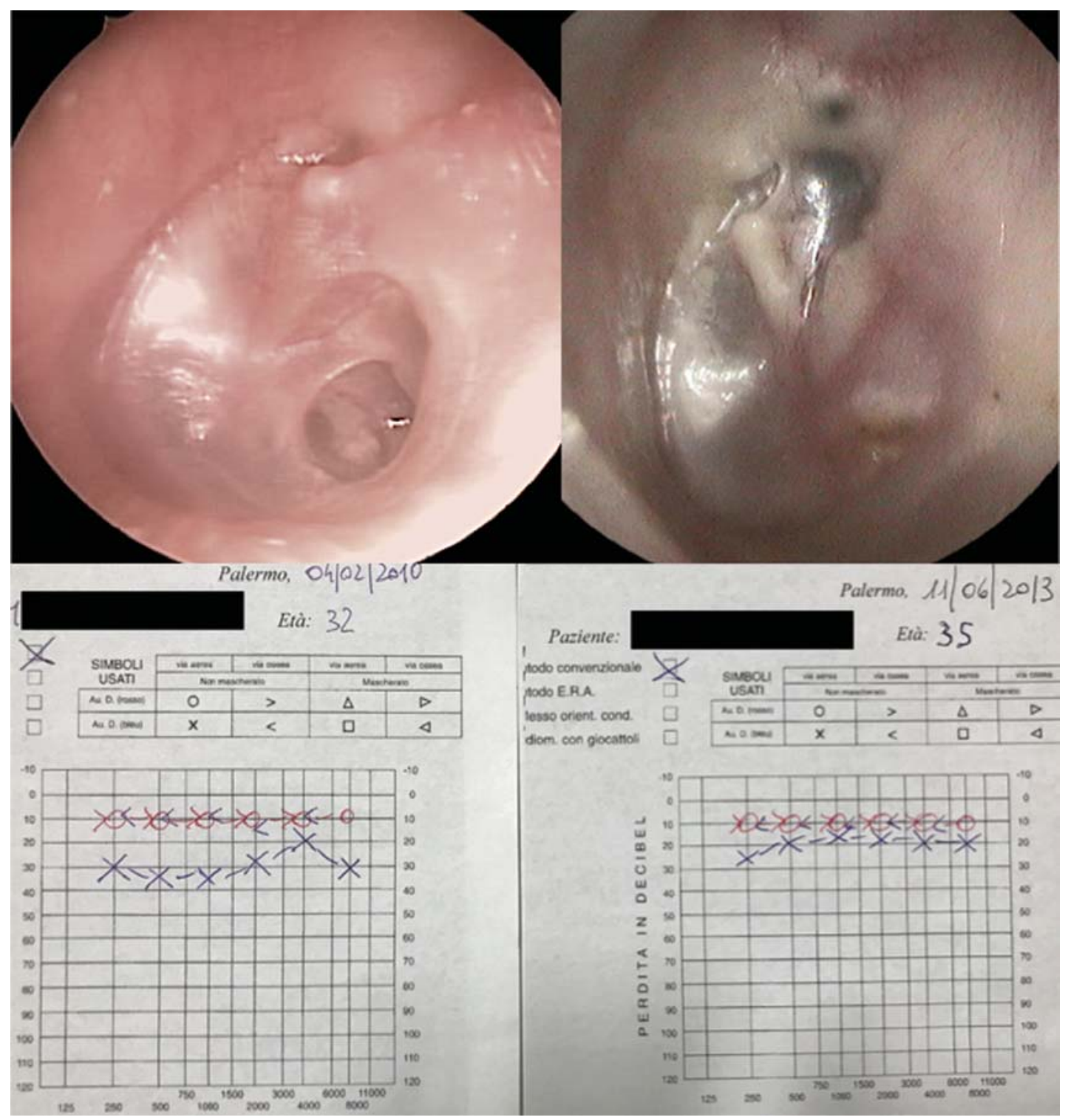

Fig. 1 Patient with posterior retraction pocket, with chain interruption, managed with reconstruction with autologous incus reshaped; otoscopy pre and postoperative with hearing threshold.

Table 3 Hearing results after surgery

\begin{tabular}{|l|l|l|l|l|}
\hline & Tympanoplasty with mastoidectomy & Tympanoplasty & $p$-value & Whole series \\
\hline Number of cases & 21 & 22 & & 43 \\
\hline ABG preoperative & $23.4 \mathrm{~dB}$ (SD 8.5) & $21.7 \mathrm{~dB}(\mathrm{SD} 5.1)$ & & $22.1 \mathrm{~dB}$ (SD 7.7) \\
\hline ABG postoperative & $6 \mathrm{~dB}(\mathrm{SD} 2.1)$ & $5.3 \mathrm{~dB}(\mathrm{SD} 1.9)$ & & $5 \mathrm{~dB}(\mathrm{SD} 1.7)$ \\
\hline Average improvement & $13.8 \mathrm{~dB}$ (SD 3.3) & $12.1 \mathrm{~dB}(\mathrm{SD} 2.6)$ & $>0.5$ & $12.5 \mathrm{~dB}$ (SD 2.6) \\
\hline Recurrence of retraction pocket & 1 & 0 & $\mathrm{NA}$ & 1 \\
\hline
\end{tabular}

Abbreviation: $\mathrm{ABG}$, air bone gap, $\mathrm{dB}$, decibels; SD, standard deviation.

tympanoplasty alone. ${ }^{15}$ Smoking, on the other hand, is a predictor of a postoperative complication, as demonstrated by their binary logistic regression.

According with a review of 2016, the addition of mastoidectomy to tympanoplasty does not improve ABG or new tympanic membrane integrity. Most of the studies included in this review included patients with perforation of the eardrum; however, our work focused on retraction pocket without perforation, but the surgical procedure "tympanoplasty" was conducted in the same way as tympanic perforation requires. ${ }^{14}$

Both Albu and McGrew, in their case-control studies based on a follow-up of 12 and 32 months, respectively, showed that there is no significant gain on practicing mastoidectomy in addition to tympanoplasty. ${ }^{16,17}$ 
Table 4 Hearing results after surgery according to ossicular chain conditions

\begin{tabular}{|l|l|l|l|}
\hline & Ossicular chain erosion & Intact ossicular chain & $p$-value \\
\hline Number of cases & 19 & 24 & \\
\hline ABG preoperative & $29.3 \mathrm{~dB}(\mathrm{SD}$ 5.3) & $12.4 \mathrm{~dB}(\mathrm{SD} 3.1)$ & \\
\hline ABG postoperative & $13.3 \mathrm{~dB}(\mathrm{SD} 3.4)$ & $0.5 \mathrm{~dB}(\mathrm{SD} 1.1)$ & \\
\hline Average improvement & $16.7 \mathrm{~dB}(\mathrm{SD} 2.5)$ & $9.3 \mathrm{~dB}(\mathrm{SD} 3.3)$ & $<0.05$ \\
\hline
\end{tabular}

Abbreviation: $A B G$, air bone gap; $\mathrm{dB}$, decibels; SD, standard deviation.

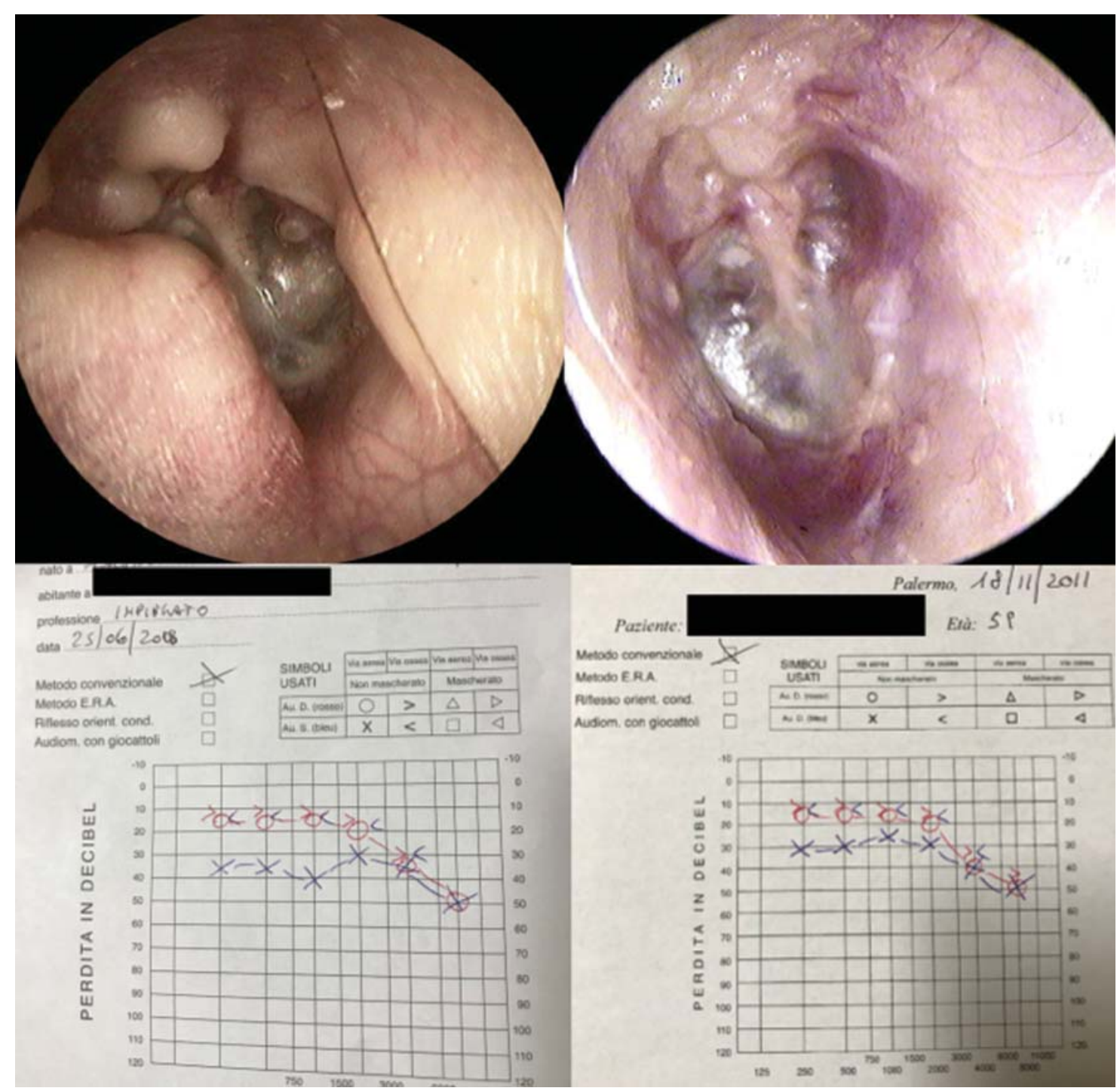

Fig. 2 Patient with external ear canal exostosis and posterior retraction pocket with ossicular chain interruption managed with canalplasty, tympanic reconstruction with fascia and cartilage, and reshaped incus as ossiculoplasty.

Even Agrawal and Bhargava, referring to the treatment of chronic suppurative otitis media tubotympanic type, compared the difference between tympanoplasty with or without mastoidectomy and concluded that mastoidectomy gives no statistically significant benefit over tympanoplasty with regards to graft success rate and hearing gain ( $p$-value is insignificant in their study). ${ }^{18}$

According to Mishiro, the most important feature to predict $A B G$ closure within a 60 -month period is an intact ossicular chain and a good quality of the eardrum graft. He prefers avoiding mastoidectomy when not strictly necessary. ${ }^{19}$

In the present study, the percentage of $A B G$ improvement was higher in the subgroup that underwent only tympanoplasty, although without statistical significance. Mastoidectomy should not be considered part of standard surgical operation for retraction pockets, but it should be used when there is high risk of hidden cholesteatoma suspicion, when 
there is an important chronic inflammation of middle ear, and when there is a recurrence of RPs after surgical treatment. ${ }^{20}$

Nevertheless, mastoidectomy can make the surgical results worse than expected (i.e., an injury to the seventh cranial nerve), and it does not often solve either tinnitus or ABG, which depend on the status of the stapes structure, and is too expensive when equating the results with the cost of surgery, so it should be done only in selected patients with specific characteristics. ${ }^{14,21}$

This study has some limitation due to the number of cases, which although enough for our population should be enlarged in time to allow for further confirmation; another limitation could be the randomization, which was simple, because the identification of all subjects of the series was not possible before group assignment being a surgical study. Consequently, another kind of randomization (i.e.: block or stratified) was not applicable.

\section{Conclusions}

Tympanoplasty and ossiculoplasty should be considered to treat hearing loss caused by atelectatic middle ear and ossicular chain interruption. Mastoidectomy does not add favorable prognostic factor to management of RP in terms of hearing recovery and recurrence rate.

\section{Conflict of Interests}

The authors declare that have no conflict of interests.

\section{References}

1 Stangerup SE, Tos M, Arnesen R, Larsen P. A cohort study of point prevalence of eardrum pathology in children and teenagers from age 5 to age 16. Eur Arch Otorhinolaryngol 1994;251(07): 399-403

2 Maw AR, Hall AJ, Pothier DD, Gregory SP, Steer CD. The prevalence of tympanic membrane and related middle ear pathology in children: a large longitudinal cohort study followed from birth to age ten. Otol Neurotol 2011;32(08):1256-1261

3 Nankivell PC, Pothier DD. Surgery for tympanic membrane retraction pockets. Cochrane Database Syst Rev 2010;(07):CD007943

4 Sadé J. Retraction pockets and attic cholesteatomas. Acta Otorhinolaryngol Belg 1980;34(01):62-84

5 Sadé J. Atelectatic tympanic membrane: histologic study. Ann Otol Rhinol Laryngol 1993;102(09):712-716

6 Borgstein J, Gerritsma TV, Wieringa MH, Bruce IA. The Erasmus atelectasis classification: proposal of a new classification for atelectasis of the middle ear in children. Laryngoscope 2007; 117(07):1255-1259
7 Cassano M, Cassano P. Retraction pockets of pars tensa in pediatric patients: clinical evolution and treatment. Int J Pediatr Otorhinolaryngol 2010;74(02):178-182

8 Charachon R, Barthez M, Lejeune JM. Spontaneous retraction pockets in chronic otitis media medical and surgical therapy. Ear Nose Throat J 1992;71(11):578-583

9 Kasbekar AV, Patel V, Rubasinghe M, Srinivasan V. The Surgical Management of Tympanic Membrane Retraction Pockets Using Cartilage Tympanoplasty. Indian J Otolaryngol Head Neck Surg 2014;66(04):449-454

10 Blaney SP, Tierney P, Bowdler DA. The surgical management of the pars tensa retraction pocket in the child-results following simple excision and ventilation tube insertion. Int J Pediatr Otorhinolaryngol 1999;50(02):133-137

11 Srinivasan V, Banhegyi G, O'Sullivan G, Sherman IW. Pars tensa retraction pockets in children: treatment by excision and ventilation tube insertion. Clin Otolaryngol Allied Sci 2000;25(04): 253-256

12 Dispenza F, Bennici E, Bianchini S, et al. Fat plug myringoplasty: Analysis of a safe procedure for small tympanic perforations. EuroMediterranean Biomedical Journal 2015;10:87-92

13 Dispenza F, Battaglia AM, Salvago P, Martines F. Determinants of Failure in the Reconstruction of the Tympanic Membrane: A Case-Control Study. Iran J Otorhinolaryngol 2018;30(101):341346

14 Trinidade A, Page JC, Dornhoffer JL. Therapeutic Mastoidectomy in the Management of Noncholesteatomatous Chronic Otitis Media: Literature Review and Cost Analysis. Otolaryngol Head Neck Surg 2016;155(06):914-922

15 Shew MA, Muelleman T, Villwock M, et al. Therapeutic Mastoidectomy Does Not Increase Postoperative Complications in the Management of the Chronic Ear. Otol Neurotol 2018;39(01): 54-58

16 McGrew BM, Jackson CG, Glasscock ME III. Impact of mastoidectomy on simple tympanic membrane perforation repair. Laryngoscope 2004;114(03):506-511

17 Albu S, Trabalzini F, Amadori M. Usefulness of cortical mastoidectomy in myringoplasty. Otol Neurotol 2012;33(04):604-609

18 Agrawal A, Bhargava P. Comparative Evaluation of Tympanoplasty with or Without Mastoidectomy in Treatment of Chronic Suppurative Otitis Media Tubotympanic Type. Indian J Otolaryngol Head Neck Surg 2017;69(02):172-175

19 Mishiro Y, Sakagami M, Kondoh K, Kitahara T, Kakutani C. Longterm outcomes after tympanoplasty with and without mastoidectomy for perforated chronic otitis media. Eur Arch Otorhinolaryngol 2009;266(06):819-822

20 Ruhl CM, Pensak ML. Role of aerating mastoidectomy in noncholesteatomatous chronic otitis media. Laryngoscope 1999;109 (12):1924-1927

21 Dispenza F, Cappello F, Kulamarva G, De Stefano A. The discovery of stapes. Acta Otorhinolaryngol Ital 2013;33(05):357-359 